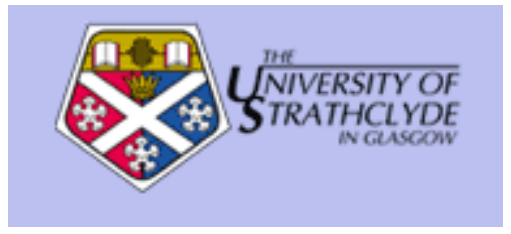

Haw, Mark D. (2010) Growth kinetics of colloidal chains and labyrinths. Physical Review E: Statistical, Nonlinear, and Soft Matter Physics, 81 (3). 031402. ISSN 1063-651X

http://strathprints.strath.ac.uk/20644/

Strathprints is designed to allow users to access the research output of the University of Strathclyde. Copyright (C) and Moral Rights for the papers on this site are retained by the individual authors and/or other copyright owners. You may not engage in further distribution of the material for any profitmaking activities or any commercial gain. You may freely distribute both the url (http://strathprints.strath.ac.uk) and the content of this paper for research or study, educational, or not-for-profit purposes without prior permission or charge. You may freely distribute the url (http://strathprints.strath.ac.uk) of the Strathprints website.

Any correspondence concerning this service should be sent to The Strathprints Administrator: eprints@cis.strath.ac.uk 


\title{
Growth kinetics of colloidal chains and labyrinths
}

\author{
Mark D. Haw * \\ Department of Chemical and Process Engineering, University of Strathclyde, James Weir Building, \\ 75 Montrose Street, Glasgow G1 1XJ, United Kingdom
}

(Received 7 August 2009; published 11 March 2010)

\begin{abstract}
Particles interacting by a combination of isotropic short-range attraction and long-range repulsion have been shown to form complex phases despite the apparent simplicity of the interparticle potential. Using computer simulations we study the behavior of two-dimensional systems of colloids with such an interaction, focusing on how area fraction and repulsion range at fixed repulsion gradient may be used to tune the resulting kinetics and nonequilibrium structure. While the short-range attraction leads to aggregation, the long-range repulsion encourages growth of chains of particles due to repulsive intercluster interactions. Depending on area fraction/ repulsion range we observe chain labyrinths, chain-compact aggregate coexistence, and connected networks of chains. The kinetics of cluster growth displays a sequence of connected networks and disconnected cluster or chain systems with increasing repulsion range, indicating the competing roles of connectivity of growing chains and repulsion-driven breakup of chains into compact aggregates. Chain-dominated systems show approximately logarithmic coarsening at late time that we interpret as the result of chains performing random walks in the randomly fluctuating potential landscape created by their neighbors, a situation reminiscent of glassy systems.
\end{abstract}

DOI: 10.1103/PhysRevE.81.031402

PACS number(s): 82.70.Gg, 81.16.Dn, 83.80.Hj

\section{INTRODUCTION}

Interactions between colloidal particles in suspension determine the structure and practical characteristics (rheology, texture, and functionality) of many important materials, including foods, composities, ceramics, cosmetics, filters, and paints [1]. Colloidal-scale interactions and their influence on structure, kinetics, and dynamics are also important in aspects of biological systems and biotechnology, such as proteins in cell membranes [2]. Developing systems with "designer" interactions to realize novel structure/kinetics/ dynamics is an important theme in colloid science and engineering [3]. The conceptually simplest interactions between colloids, namely, purely attractive or purely repulsive isotropic potentials, have been studied extensively in experiments, simulations and as part of theories of colloidal stability [1]. Recently exploration of the possible structural and kinetic behavior in systems of particles with somewhat more complex interactions, such as isotropic potentials involving soft repulsion around a hard core, or attraction and repulsion at different ranges, has revealed surprising complexity in structure and phase behavior, such as repulsion-driven clustering, chain formation, and complex network structures [4].

In this paper we use two-dimensional (2D) computer simulations (with relevance to 2D systems such as particleladen films, deposition, surface patterning, and particles confined in membranes) to investigate a system of colloidal particles interacting with a combination of short-range attractive potential at particle separation of a small fraction of the particle radius, and a long-range repulsive potential. We focus on how both the structural and kinetic features can be tuned by variation of particle area fraction and repulsion range. In contrast to most previous investigations we focus on the ki- netics of growth of structures such as chains and aggregates, rather than the equilibrium or minimum energy structures [4]. Briefly, the short-range attraction leads to clustering of particles as expected; however the long-range repulsive barrier has significant consequences, including, as observed in other similar models, the formation of colloidal chains, labyrinthine chain networks, and coexisting chain/aggregate structures. We explore how kinetic properties, rates of chain growth etc. vary with system parameters. We demonstrate how a sequence of connected chain networks appears and disappears as repulsion range is increased, implying that fine tuning of repulsion may provide a sensitive switch for connectivity of the system. We argue that nonequilibrium structures such as connected chain "gels" are practically important since the kinetic barriers to equilibrium are significant: in the chain-dominated systems especially we see an approximately logarithmic coarsening in time, reminiscent of glassy systems with a very slow approach to equilibrium [5].

\section{METHODS}

We use a diffusing particle simulation to explore the structural evolution of a 2D system of $N$ particles (circular disks), interacting with a potential $E(\delta)$ where $\delta$ is the interparticle separation. The $N$ particles are placed at random in a square simulation box of size $L$ with periodic boundary conditions in $x$ and $y$ directions, giving an area fraction $\Phi$ $=N\left(\pi r^{2}\right) / L$ where $r$ is the particle radius. The particles diffuse by short random jumps. Move attempts are generated with random components in the $x$ and $y$ directions selected from a uniform distribution with maximum $d=0.005 r$. The potential energies of the particle at its initial and final (postmove) positions are calculated from the sum of interparticle potentials of the particle's neighbors. Attempted moves are accepted according to a probability $P=\exp (-\Delta E)$ where $\Delta E$ is the potential energy change associated with the attempted 


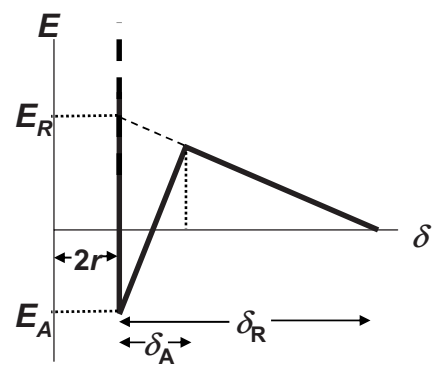

FIG. 1. The interparticle potential $E$ used in the simulations, vs particle separation $\delta . E_{A}$ is the attractive energy at touching (particle separation $\delta=2 r$ ), $\delta_{A}$ is the range of the attractive potential well (measured from touching); $E_{R}$ is the repulsive energy (extrapolated to separation $\delta=2 r$; for repulsive gradient studied here the height of the repulsive barrier at $\delta_{A}$ is equal to $\left.E_{R}-0.375\right) ; \delta_{R}$ is the range beyond which the interparticle energy is zero.

move, in units of the thermal energy $k_{B} T$. (Moves with $P>1$, i.e., decreasing energy, are always accepted.) Overlaps between particles lead to rejection of moves, i.e., the particle "core" is a "hard disk." This method of simulation is appropriate for systems such as colloids where dynamics is dominated by Brownian fluctuations, although studies of for instance minimum energy configurations with similar interactions [4] show that results are insensitive to simulation methods. We have also checked that the structures observed do not depend on the initial random configuration of particles, i.e. repeat runs with new random initial configurations give statistically similar results.

The interparticle potential we study here has the 'linear ramp' shape shown in Fig. 1. The potential is defined by 4 parameters: the interparticle attraction $E_{A}$ at "touching" (i.e., attraction energy at zero particle surface separation $\delta=2 r$ ), the range of the attractive well $\delta_{A}$ (measured between particle surfaces, see Fig. 1), the repulsion energy $E_{R}$ and the range of the repulsive potential $\delta_{R}$ (beyond which $E=0$ ). In these results we fix $E_{A}=-7 k_{B} T, \delta_{A}=0.05 r$, and consider constant gradient of the repulsive potential, $E_{R} / \delta_{R}=7.5 k_{B} T$ per unit particle radius. For convenience we give the repulsive energy $E_{R}$ extrapolated to $\delta=2 r$ as shown in Fig. 1 . We use a triangular repulsive potential between $\delta_{A}$ and $\delta_{R}$, and a triangular attractive well for $\delta<\delta_{A}$ (note that we find no significant changes for a square well attraction).

In the results presented here we consider repulsive energies/ranges between $\left\{E_{R}=3.75 k_{B} T, \delta_{R}=0.5 r\right\}$ to $\left\{E_{R}\right.$ $\left.=60 k_{B} T, \delta_{R}=8.0 r\right\}$. For the constant repulsive gradient used here the height of the repulsive barrier at $\delta=\delta_{A}$ is equal to $E_{R}-0.375 k_{B} T$. Simulations are carried out across a range of $\Phi$ from 0.1 to 0.5 . Typical runs are performed with $N$ $=1000$ particles over total times on the order of $t=10^{7}$ timesteps, where a timestep represents 1 attempted move per particle. A range of runs has also been carried out with $N$ $=4000$ and we find no change in observed structure or kinetics, i.e., finite size effects are negligible. We have also checked that the results are insensitive to the maximum step length $d$ as long as $d<0.01 r$. For convenience and generality we report time scales in numbers of simulation steps: to compare with real colloidal systems, we note that the "diffusion time," i.e., the time taken for a single isolated particle to

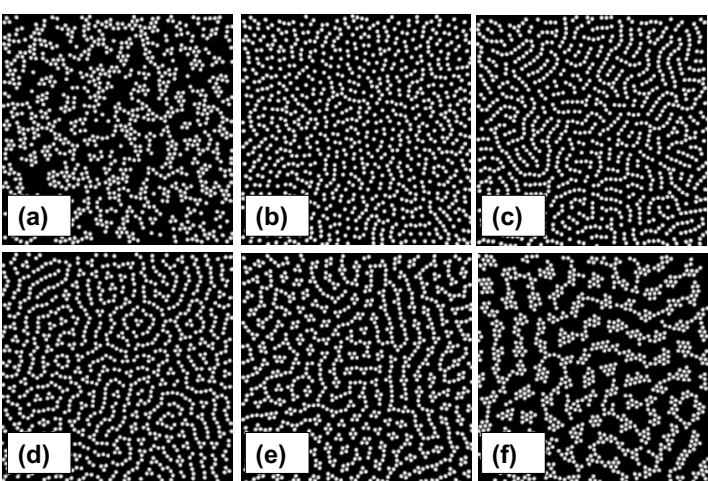

FIG. 2. Dependence of system structure on repulsive energy and range $\left\{E_{R}, \delta_{R}\right\}$ at area fraction $\Phi=0.35$, where $E_{R}$ is in units of $k_{B} T$ and $\delta_{R}$ is in particle radii. (a) $\left\{E_{R}=3.75, \delta_{R}=0.5\right\}$; (b) $\left\{E_{R}\right.$ $\left.=11.25, \delta_{R}=1.5\right\}$; (c) $\left\{E_{R}=15, \delta_{R}=2.0\right\}$; (d) $\left\{E_{R}=22.5, \delta_{R}=3.0\right\}$; (e) $\left\{E_{R}=30, \delta_{R}=4.0\right\}$; and (f) $\left\{E_{R}=60, \delta_{R}=8.0\right\}$. Each snapshot shows the particle configurations at $t=10^{7}$ timesteps.

diffuse its radius, with the given maximum step length $d$, is $\sim 4 \times 10^{5}$ steps; the diffusion time scale for a colloid of radius $500 \mathrm{~nm}$ in a solvent with viscosity $1 \times 10^{-3} \mathrm{~Pa} \mathrm{~s}$ at temperature $293 \mathrm{~K}$ is, according to the Stokes-Einstein relation, $\sim 25 \times 10^{-3}$ s. Hence, the longest time scales reported here $\left(\sim 10^{7}\right.$ steps $)$ represent times on the order of $1 \mathrm{~s}$ for typical colloidal systems.

\section{RESULTS AND DISCUSSION}

Example snapshots from simulations at fixed $\Phi=0.35$ for varying repulsion $\left\{E_{R}, \delta_{R}\right\}$ are shown in Fig. 2. Figures 3 and 4 compare systems across $\Phi$, for fixed repulsion energies/ ranges $\left\{E_{R}=15 k_{B} T, \delta_{R}=2.0 r\right\}$ and $\left\{E_{R}=30 k_{B} T, \delta_{R}=4.0 r\right\}$, respectively. In Fig. 5 we plot a structural diagram $\left(\Phi\right.$ vs $\left.\delta_{R}\right)$
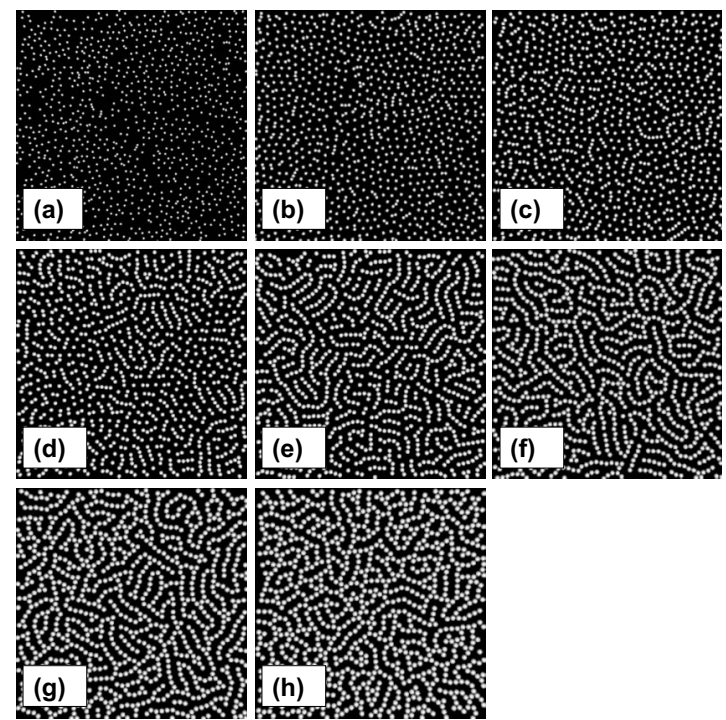

FIG. 3. Dependence of system structure on area fraction $\Phi$, at fixed repulsion $\left\{E_{R}=15 k_{B} T, \quad \delta_{R}=2.0 r\right\}$. (a) $\Phi=0.1$; (b) $\Phi=0.2$; (c) $\Phi=0.25$; (d) $\Phi=0.3$; (e) $\Phi=0.35$; (f) $\Phi=0.4$; (g) $\Phi=0.45$; and (h) $\Phi=0.5$. Each snapshot shows the particle configurations at $t=10^{7}$ timesteps. 


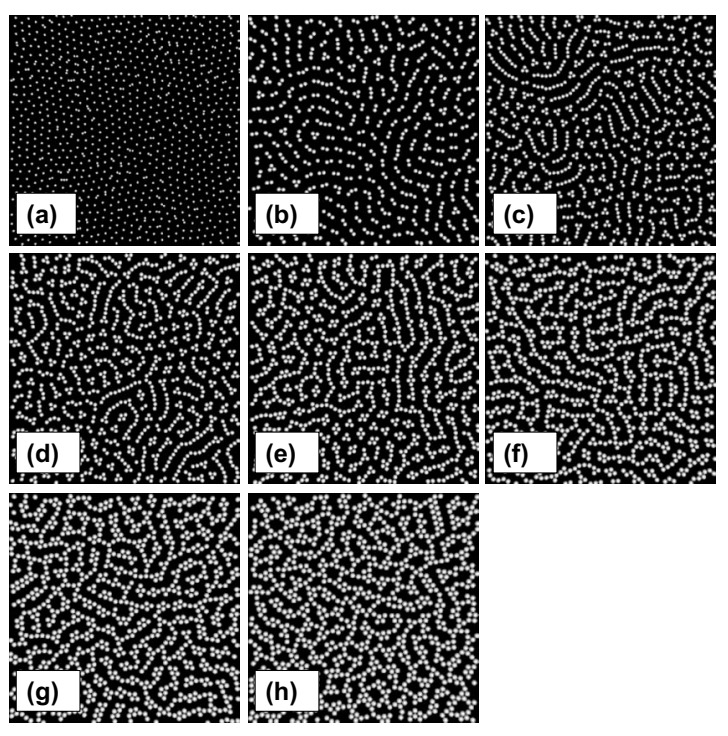

FIG. 4. Dependence of system structure on area fraction $\Phi$, at fixed repulsion $\left\{E_{R}=30 k_{B} T, \delta_{R}=4.0 r\right\}$. (a) $\Phi=0.1$; (b) $\Phi=0.2$; (c) $\Phi=0.25$; (d) $\Phi=0.3$; (e) $\Phi=0.35$; (f) $\Phi=0.4$; (g) $\Phi=0.45$; and (h) $\Phi=0.5$. Each snapshot shows the particle configurations at $t=10^{7}$ timesteps.

based on the structures observed: hence the snapshots in Fig. 2 follow a vertical line up the diagram demonstrating the change in system structure with repulsion energy/range at constant $\Phi$, while Figs. 3 and 4 follow horizontal lines across the diagram demonstrating the dependence of system structure on $\Phi$ at constant $\left\{E_{R}, \delta_{R}\right\}$.

As can be seen even the relatively simple extension of interparticle potential from purely attractive or repulsive to combined short-range attraction/long-range repulsion leads to a rich structural behavior [4]. We can identify various regions of structure from Fig. 5 and the snapshots, including:

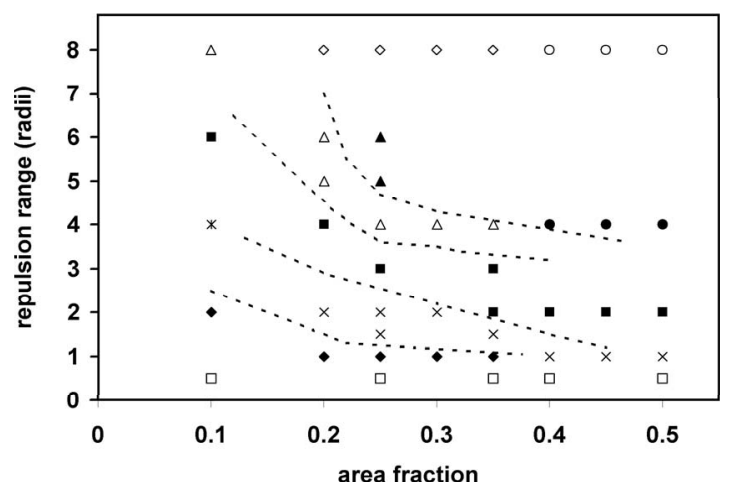

FIG. 5. System "structural" diagram showing approximate regions of area fraction $\Phi$ and repulsion range $\delta_{R}$ typified by various structures, as described in the text. Dashed lines are guides to the eye. Symbols: open squares=aggregation; filled diamonds = disordered monomers (plus occasional chain); crosses $=$ monomer + chains mixture; filled squares=chain labyrinth; open triangles=chain $/$ blob coexistence; filled triangles $=$ blobs + "knotty" chains mixture; filled circles=blobs + 'knotty chain' network; open diamonds=large, bridged irregular blobs; open circles $=$ large, irregular blob network (i.e., fully bridged). (a)

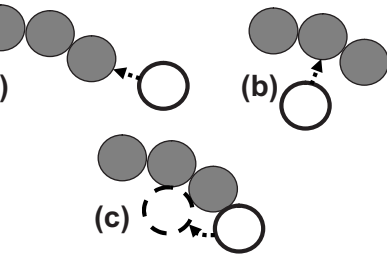

FIG. 6. Chain growth and chain compactification. (a),(b): for an isolated cluster, the long-range repulsion encourages endattachment (a) as opposed to attachment near the middle of the cluster (b), because this minimizes repulsion by neighbors further along the chain. (c) For nonrigid bonds, an end-attaching particle can roll around its end-neighbor at low energy cost (remaining within the attractive potential of the end-neighbor). The repulsive energy barrier to bonding with the next neighbor in the chain, and thus compactifying the chain, is the same as the energy barrier to a new monomer attaching to the chain end: thus the compactification rate is at least as fast as the chain growth (end-attachment) rate, and aggregates grow compactly, not as chains.

aggregation without chains [Fig. 2(a)]; coexistence of monomers and chains [Figs. 2(b), 3(b), and 3(c)]; "labyrinths" of chains, both disconnected [Figs. 2(c), 2(d), 3(e), 3(f), and 4(b)] and connected in a "chain network" [Figs. 3(g), 3(h), 4(g), and 4(h)]; chain/"blob" (i.e. compact aggregate) coexistence [Figs. 2(e) and 4(c)-4(e)]; and larger, loosely spatially ordered, irregular-shaped blobs often "bridged" by connecting chains [Fig. 2(f)].

The most basic question we can ask concerns chain formation: why should increasing $\Phi$ and $\left\{E_{R}, \delta_{R}\right\}$ lead to a transition between aggregation and growth of chains? The case of colloidal particles aggregating under a purely attractive short-range potential has been extensively studied [6]. For rigid bonds, it is well known and confirmed experimentally that fractal aggregates of particles are formed and ultimately, if aggregation continues for long enough, growing fractal aggregates eventually span the system to form a particle gel. However at first sight the combination of short-range attraction and long-range repulsion would indeed seem likely to encourage chains as opposed to aggregates. A monomer attaching to an existing (small) chain (e.g. a linear trimer) feels minimum repulsion by attaching to the end of the existing chain so that the other members of the chain are maximally far away [Figs. 6(a) and 6(b)]. The long-range repulsion therefore encourages aggregates to grow by end-addition of monomers, i.e., to grow as linear chains.

However, further consideration shows that the above argument for chain formation is too simplistic. Consider a monomer that has attached to the end of a small chain. Unless bonding is rigid, once within the range of the attractive potential the newly attached monomer may "roll" (without significant change in interparticle separation and thus at minimal energy cost) around the original end particle. Eventually the rolling particle may attach to the next particle in the chain, leading to compactification of the chain [Fig. 6(c)]. Of course, due to the repulsive part of the potential there is a significant energy barrier to the rolling particle bonding with the next neighbor and driving compactification in this way: but this energy barrier to compaction is approximately the same as the barrier to another new monomer 
attaching to the end of the chain [7]. Hence, the compaction rate of small chains is at least as fast (or faster) than addition of further monomers to the chain: the chain compacts faster than it grows.

This argument considers growth of an isolated aggregate or chain. When clusters grow essentially independently therefore, i.e. at low $\Phi$ and/or low $\left\{E_{R}, \delta_{R}\right\}$ where growing chains are outside the range of repulsion of any neighboring chains, we expect no tendency toward chain formation. This is consistent with our observations at the lowest repulsion range and at low enough $\Phi$ that aggregates have space to grow independently and, hence, compactify faster than chain formation: we see straightforward aggregation [Fig. 2(a)].

So why should chains form at all when bonding is isotropic and not rigid and if chains compactify faster than they grow? The key point is that at higher $\Phi$ and repulsion range aggregates do not grow independently: they are always subject to repulsive interactions with their neighbors (whether other clusters or monomers). Thus stable noncompactifying chains can form due to the fact that at higher $\left\{E_{R}, \delta_{R}\right\}$ or $\Phi$, intercluster interactions become important. The potential still leads to preferential end attachment and, thus, growth of small chains. But now these growing chains are stopped from compactifying by the repulsive interaction of neighboring particles or other aggregates/chains, and chain growth thus continues. For example at low $\left\{E_{R}, \delta_{R}\right\}$ we see the onset of chain formation [Figs. 3(b) and 3(c)] within a sea of monomers, whose collective repulsion is enough to stabilize the chains against compaction. As $\left\{E_{R}, \delta_{R}\right\}$ or $\Phi$ increases more particles form chains, and interchain repulsion becomes significant. Interchain repulsion, as opposed to stabilization of an isolated chain by surrounding monomers, leads to a further structural development, i.e., significant local nematic alignment of chains, both parallel and "end-on." This ultimately results in the formation of winding chain labyrinths [Figs. 2(c), 2(d), 3(e)-3(h), and 4(b)]. The typical length of chains in the labyrinth increases with $\Phi$ : compare Figs. 3(e) and 4(b), labyrinths at $\Phi=0.35$ and 0.2 , respectively.

What happens to chains as we further increase repulsion energy/range? Figures 2(c) and 2(d) show that chains in the labyrinth become increasingly irregular and sharply "kinked" due to the necessity for particles to minimize energy in the landscape created by longer-range repulsion. In fact systems demonstrate distinct behavior depending on position in the $\Phi-\left\{E_{R}, \delta_{R}\right\}$ diagram. In Fig. 2(e), $\Phi=0.35$, as repulsion range increases some chains are replaced by small "blobs" (compact aggregates) that coexist with remaining chains. The remaining chains have a "knotty" appearance and could also be thought of as small blobs (e.g., compact trimers) connected by chains. The fraction of chains vs blobs depends on $\left\{E_{R}, \delta_{R}\right\}$, implying that varying the interaction can provide fine control of the structural details of the system. Finally, as repulsion range increases further, larger irregular-shaped aggregates with a rough spatial ordering dominate. These larger aggregates are sometimes nevertheless bridged by remaining chains [Fig. 2(f)], i.e., traces of chain formation remain, and at the highest $\Phi$ a fully bridged network of irregular-shaped large blobs appears (open circles in Fig. 5).
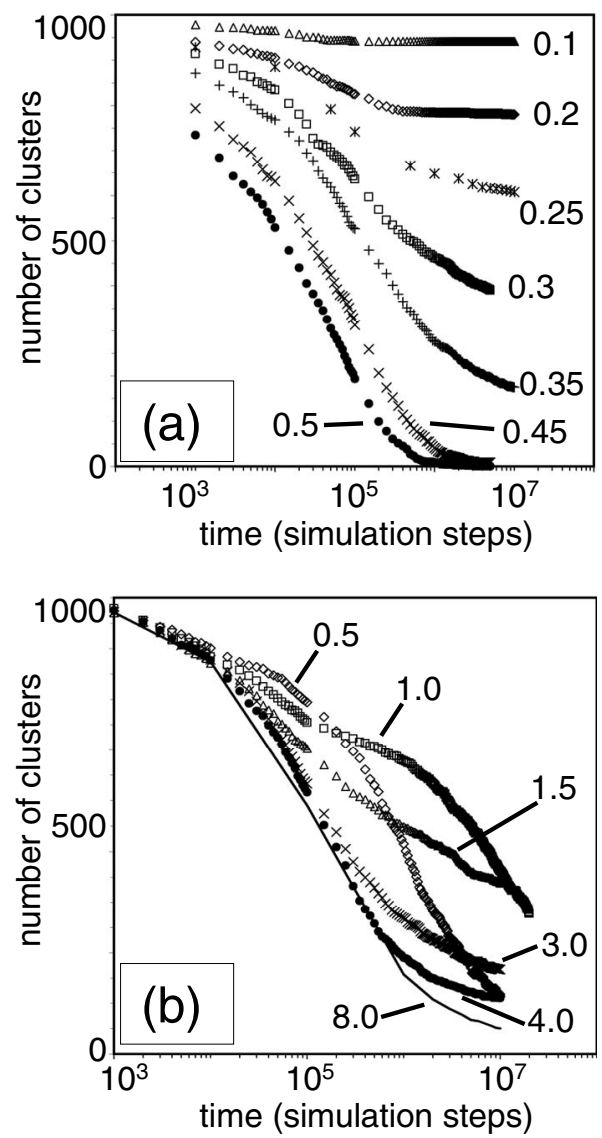

FIG. 7. Kinetics of aggregate/chain growth. The number of clusters of particles is plotted vs simulation timestep, where particles separated by less than the attractive potential range $\delta_{A}$ are considered part of the same cluster. (a) $\left\{E_{R}=15, \delta_{R}=2.0\right\}$, where $E_{R}$ is in units of $k_{B} T$ and $\delta_{R}$ is in particle radii. The labels for each curve give the area fraction $\Phi$. (b) Comparison of different $\left\{E_{R}, \delta_{R}\right\}$ at area fraction $\Phi=0.35$; the labels give the value of repulsion range $\delta_{R}$ in particle radii.

Figures 3 and 4 show how system behavior depends on $\Phi$ at fixed repulsion, i.e. how, with fixed particle interaction, we can tune system structure by varying $\Phi$. With $\left\{E_{R}\right.$ $=15 k_{B} T, \delta_{R}=2.0 r$ (Fig. 3) we see a progressive increase in chain population with $\Phi$, until the entire system is composed of chains and a chain labyrinth results. At this repulsion, in contrast to the discussion in the previous paragraph, we do not observe the chain labyrinth breaking up into blob/chain coexistence up to the highest $\Phi$ studied (0.5). Rather increasing $\Phi$ results in an ever more compressed chain labyrinth [Figs. 3(f)-3(h)]. Hence, changing $\Phi$ at lower $\left\{E_{R}, \delta_{R}\right\}$ allows tuning of the density of the chain labyrinth or network, another "control parameter" on the system structure.

To obtain more insight into the system behavior we consider cluster growth kinetics. Figure 7 shows the number of separate clusters $N_{c}$ vs timestep, where particles within the attraction range $\delta_{A}$ are considered as belonging to the same cluster. Figure 7(a) contrasts kinetic behavior at different $\Phi$, for fixed repulsion $\left\{E_{R}=15 k_{B} T, \delta_{R}=2 r\right\}$. Below $\Phi \sim 0.35$ growth freezes at late time as monomers and small chains find a stable configuration in the energy landscape. Figure $7(\mathrm{~b})$, showing dependence of kinetics on $\left\{E_{R}, \delta_{R}\right\}$ at fixed 

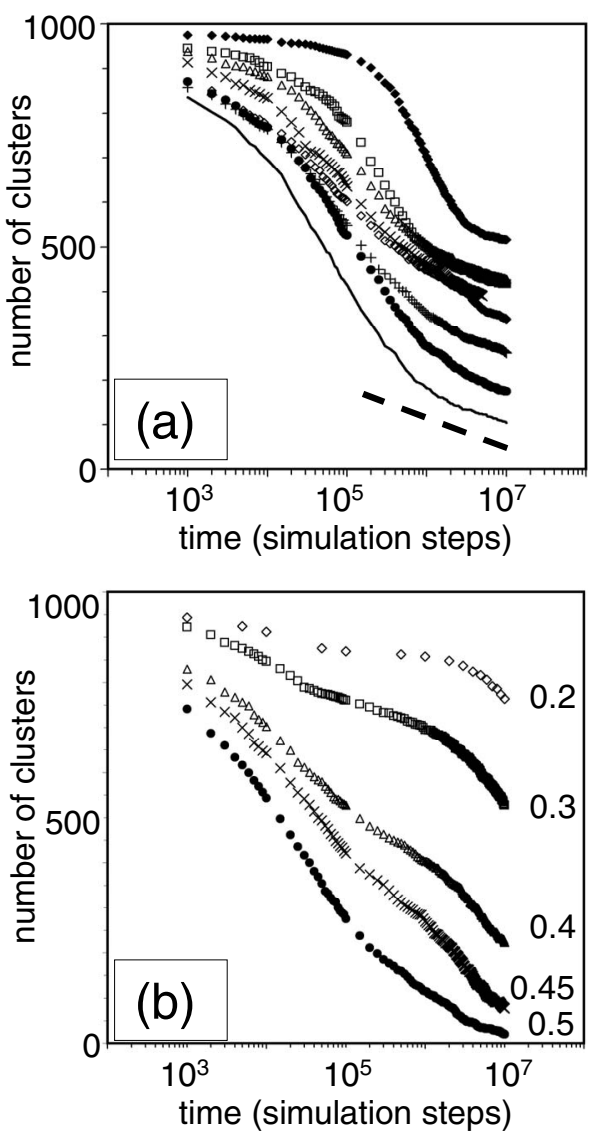

FIG. 8. (a) Number of clusters $N_{c}$ vs timestep for systems where chains are the dominant structure. At long time, decay of $N_{c}$ becomes approximately logarithmic in time as demonstrated by the short dashed straight line at the bottom of the figure. Symbols from top to bottom curve as follows: filled diamonds, $\Phi=0.1,\left\{E_{R}\right.$ $\left.=45, \delta_{R}=6.0\right\}$; open squares $\Phi=0.2,\left(E_{R}=30, \delta_{R}=4.0\right)$; open triangles, $\Phi=0.25, \quad\left\{E_{R}=22.5, \delta_{R}=3.0\right\} ; \quad$ crosses, $\Phi=0.3, \quad\left\{E_{R}\right.$ $\left.=15, \delta_{R}=2.0\right\}$; open diamonds, $\Phi=0.35,\left\{E_{R}=11.25, \delta_{R}=1.5\right\}$; pluses, $\Phi=0.35,\left\{E_{R}=13.175, \delta_{R}=1.75\right\}$; filled circles, $\Phi=0.35$, $\left\{E_{R}=15, \quad \delta_{R}=2.0\right\} ;$ continuous line, $\Phi=0.4, \quad\left\{E_{R}=13.175, \quad \delta_{R}\right.$ $=1.75\}$. (b) Number of clusters vs timestep for repulsion $E_{R}$ $=3.75 k_{B} T, \delta_{R}=0.5$ radii, where continuing growth (aggregation) of irregular clusters dominates. The labels for each curve give area fraction $\Phi$.

$\Phi=0.35$, reveals different growth regimes as repulsion energy and range increases: from an initially slow, but at late time rapid and continuing aggregation regime at low $\left\{E_{R}, \delta_{R}\right\}$, where chains are not observed [compare Fig. 2(a)], to a regime of initially faster growth, corresponding to the appearance of chains of particles as $\left\{E_{R}, \delta_{R}\right\}$ increases, followed by slower late-time evolution.

Figure 8 contrasts the kinetic behavior in chain-dominated systems [Fig. 8(a)] with that in systems at low repulsion energy/range [Fig. 8(b)]. In the low repulsion range regime, growth accelerates at long time, i.e., aggregation ultimately dominates. Large irregular clusters become increasingly likely to meet, while because the repulsion is short range the effective repulsive barrier to aggregating remains unchanged: hence, continuing aggregation dominates. In contrast, growth in the chain-dominated, longer-range repulsion systems slows at long time to an approximately logarithmic decay of $N_{c}$ [dashed line in Fig. 8(a)]. Here intercluster repulsion between chains is significant. The logarithmic decay of the number of chains can be seen as a consequence of chains carrying out random walks in the randomly fluctuating energy landscape created by their (also random walking) neighbors [8]. Very slow fluctuations leading to a logarithmic aging or coarsening invites comparison with glassy dynamics [5]: it is tempting to view the labyrinth as a glass of chains. Confirmation of "true" glassy behavior requires detailed study of dynamics, which we have not attempted here.

Many previous studies have focused on the question of the equilibrium phases or minimum energy configurations of systems of particles with similar interactions [4]. Here, we focus on kinetics of approach to (potentially "distant") equilibrium. While the structures we observe may not be at global equilibrium, Figs. 7 and 8 demonstrate that the ongoing late-time kinetics and structural evolution are very slow, in all cases apart from aggregation-dominated systems (i.e., at low-repulsion energy/range). Chains are embedded in the fluctuating but very slowly evolving (if at all) energy landscape created by their neighbors, and large (compared to thermal energy $k_{B} T$ ) barriers prevent repulsive clusters or chains coalescing. The system "explores" possible configurations slowly: chains do locally fluctuate, but large-scale evolution is much slower. For practical purposes, we would argue that it is not so much the equilibrium phases as the kinetically favored nonequilibrium behavior of these systems that is important. The nonequilibrium behavior is commonly of most interest in practical applications of colloids since interparticle interactions are weak compared to driving forces, yet kinetic barriers are high compared to thermal fluctuations. Put simply, it is easy to drive a colloidal system out of equilibrium, but difficult for thermal forces to return it to equilibrium $[1,6]$.

Figure 9 shows the dependence of the size (number of particles) of the largest connected cluster $m_{l}$, at fixed late time, on repulsion range $\delta_{R}$, for fixed $\Phi=0.4$. (By this late time further system evolution has slowed to become almost negligible.) The plot reveals a surprising kinetic/structural complexity. Increasing $\delta_{R}$ we see a sequence of connectivity/ disconnection "transitions." Increasing repulsion range from $1.5 r$ to $\sim 2 r$ results in a marked increase in $m_{l}$. [While the largest cluster size is subject to some statistical variation from run to run of the simulation, the inset to Fig. 9 shows that the more robust root mean square (rms) mass of clusters in the system at the given time, $m_{r m s}$, also shows the same pattern of peaks, and that the peaks are robust across repeat independent runs.] The fast increase of $m_{l}$ and $m_{r m s}$ indicates the formation of a large connected labyrinth of chains spanning the system, the onset of a chain network or chain "gel," as shown in the left-hand snapshot in Fig. 9. Such a spanning network will clearly have significantly different physical properties, e.g., conductivity and force response, compared to the disconnected system of chains at slightly lower $\delta_{R}$ : fine tuning of repulsion range may, therefore, offer significant control of physical properties that are dependent on connectivity.

However, the system behavior is yet more complex. Increasing $\delta_{R}$ again to $\sim 2.5 r$ we now see a marked drop in $m_{l}$ 


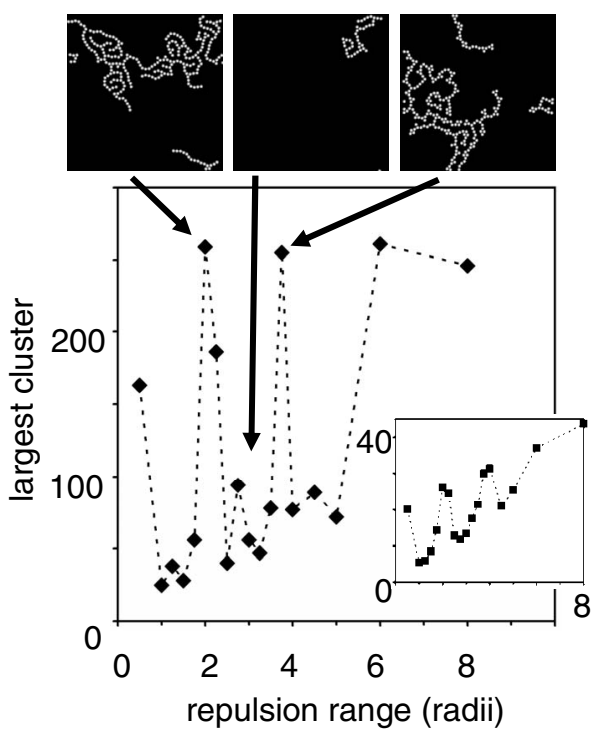

FIG. 9. Main plot: dependence of the size (number of particles) of the largest cluster in the system $m_{l}$ on repulsion range $\delta_{R}$ at fixed $t=5 \times 10^{6}$ timesteps and fixed $\Phi=0.4$, for a set of typical runs. Snapshots at the top of the figure show the structure of the largest cluster in the system for the $\delta_{R}$ indicated by the arrows: left-hand picture, $\delta_{R}=2.0$; middle, $\delta_{R}=2.5$; right-hand, $\delta_{R}=3.75$. Inset: the root mean square cluster mass $m_{r m s}$ vs $\delta_{R}$ calculated from all clusters in the system at the same timestep, then averaged over six independent repeat runs at each $\delta_{R}$.

and $m_{r m s}$ : connectivity has been lost by a slight further increase in repulsion range (middle snapshot in Fig. 9). This is explained by the increased population of small compact blobs, energetically favored by the slight change in $\delta_{R}$, replacing some chains and thus breaking up the connected chain network. Increasing $\delta_{R}$ slightly again, we see the formation of yet another connected chain network (right-hand snapshot in Fig. 9). Further increase of $\delta_{R}$ yet again "destroys" this connected network as larger compact blobs replace more chains. Possibly this sequence of connected network - disconnected chains/blobs — connected networkdisconnected chains/blobs is repeated as $\delta_{R}$ is increased further, though it becomes difficult to resolve with the set of repulsion ranges we have studied in detail so far. The question arises whether this is a true sequence of percolation/ depercolation transitions with $\delta_{R}$, or whether there is a single critical $\delta_{R}$ above which a sufficiently large system at sufficiently long time (where this time may depend on $\delta_{R}$ ) will always contain a percolating network: if it is really a sequence, it implies that small changes in repulsion range can be used to switch between (different) large-scale connected gel structures and disconnected "sols" of chains and blobs. Further "higher resolution" examination of behavior with $\delta_{R}$, scaling analysis with system size to look at the percolation behavior, and more detailed examination of the structural changes associated with the sequence of connection and disconnection (compare the structure of spanning clusters in left-hand and right-hand snapshots in Fig. 9), will be reported elsewhere.

Finally we point out that the connected chain network or gel has very different structural properties to a gel formed by attractive interactions alone [as would form here for, e.g., $\left\{E_{R}=3.75 k_{B} T, \delta_{R}=0.5 r\right\}$ at $\Phi=0.35$, Fig. 2(a)]. The latter type of gel has been studied in some detail in the context of colloidal aggregation [6]: as already mentioned space filling in that case occurs because fractal clusters are formed by aggregating particles. In the system studied here, in contrast, space filling and connectivity occurs because of the growth of connected chains, stabilized by the repulsive interchain interaction. It would be interesting to compare the mechanical response of a chain gel, which may have relevance for example to the case of particles embedded in membranes subject to external forces, to that of gels and percolating systems of different structure and origin.

\section{CONCLUSIONS}

We have explored how even a relatively simple extension to a colloidal interparticle interaction, adding a long-range repulsion to a short-range attraction, leads to a rich (nonequilibrium) structural and kinetic system behavior. In the 2D case studied here relevant applications include particles trapped in liquid-liquid films or at liquid-gas interfaces, particles in membranes, deposition of thin films and coatings, design of optical, and physical filters, structural behavior of species in cell membranes, and so on. Our results indicate that design of real colloidal systems with similar attractive +repulsive interactions (e.g., using screened Coulomb interactions to generate long-range repulsion, where salt concentration can be used to tune the repulsion range by screening interparticle repulsion, plus depletion of nonadsorbing polymers to provide similarly tunable short-range attraction) could open up a range of structures and nonequilibrium kinetics amenable to fine-tuned control, with novel uses in functional materials applications. In ongoing work we are studying interchain energetics to quantify how dynamics, kinetics, and local structure depend in detail on blob and chain shapes and configurations.
[1] W. B. Russel, D. A. Saville, and W. R. Schowalter, Colloidal Dispersions (Cambridge University Press, Cambridge, England, 1989); R. G. Larson, The Structure and Rheology of Colloidal Dispersions, (Oxford University Press, London, 1999); R. A. L. Jones, Soft Condensed Matter (Oxford University Press, Oxford, 2002).
[2] See, e.g., Protein-Lipid Interactions: From Membrane Domains to Cellular Networks, edited by L. K. Tamm (Wiley$\mathrm{VCH}$, Dordrecht, 2005).

[3] S. Torquato Soft Matter 5, 1157 (2009); M. Rechtsman, F. Stillinger, and S. Torquato, Phys. Rev. E 73, 011406 (2006).

[4] P. C. Hemmer and G. Stell, Phys. Rev. Lett. 24, 1284 (1970); 
E. Jagla, J. Chem. Phys. 110, 451 (1999); G. Malescio and G. Pellicane, Nature Mater. 2, 97 (2003); P. J. Camp, Phys. Rev. E 68, 061506 (2003); G. Malescio and G. Pellicane, ibid. 70, 021202 (2004); P. J. Camp, ibid. 71, 031507 (2005); N. Osterman et al., Phys. Rev. Lett. 99, 248301 (2007); A. J. Archer and N. B. Wilding, Phys. Rev. E 76, 031501(2007); J. Dobnikar, J. Fornleitner, and G. Kahl, J. Phys.: Condens. Matter 20, 494220 (2008); G. J. Pauschenwein and G. Kahl, J. Chem. Phys. 129, 174107 (2008); J. Fornleitner and G. Kahl, EPL 82, 18001 (2008); A. J. Archer, Phys. Rev. E 78, 031402 (2008).

[5] F. Sciortino and P. Tartaglia, Adv. Phys. 54, 471 (2005); O. Henrich et al., Phys. Rev. E 76, 031404 (2007); G. G.
Kenning, G. F. Rodriguez and R. Orbach, Phys. Rev. Lett. 97, 057201 (2006).

[6] See, e.g., M. Laurati et al., J. Chem. Phys. 130, 134907(2009); W. C. K. Poon, J. Phys.: Condens. Matter 14, R859 (2002); W. C. K. Poon and M. D. Haw, Adv. Colloid Interface Sci. 73, 71 (1997).

[7] In fact the effective rate of this "compaction" is faster because the compacting particle is bound to the end particle, hence its "attempt rate" to cross the repulsive barrier of the second neighbour and bond with it is higher.

[8] F. Krzyzewski and M. A. Zaluska-Kotur, Phys. Rev. E 77, 031502 (2008). 